\title{
Consultation in Applied Behavior Analysis: Making Least Restrictive Most Effective
}

\section{Rachel Sari*}

Brett DiNovi \& Associates, LLC, Mt. Laurel, NJ, USA

Keywords: Applied behavior analysis; Consultation

\section{Introduction}

As both a science and a practice, Applied Behaviour Analysis (ABA) is relatively young. The roots of this treatment for Autism Spectrum Disorders and myriad other conditions reach back to the work of B.F. Skinner's work circa 1938 when he published his first book, The Behaviour of Organisms. However, in less than a century, Applied Behaviour Analysts have evolved out of Behaviourism into a distinct group of scientists with a unique mission to assess and change behaviour that is socially valid to the client through the use of empirically validated methods.

In a short time, ABA established itself as a science, grew a following, compiled peer-reviewed studies and replications of such to support its validity, became a profession with its own credentials, developed national and international professional organizations holding conferences to display new research and practices, and has even further evolved to include four different levels of certification, licensure in some states, and a system of continuing education and supervision for those who practice this complex and crucial science. The title Board Certified Behaviour Analyst (BCBA), which just 20 years ago did not exist, is now in such high demand that in some areas, BCBAs face challenges of ever growing caseloads and understaffing.

Despite the explosion of ABA into the scientific and psychological communities, behaviour analysts have managed to develop a wide array of disciplines in which the science is effectively used. These include, but are not limited to, organizational behaviour management, animal behaviour, addiction and substance abuse, and of course, Autism Spectrum Disorder and related developmental disabilities. The commonality lays in the science's very name: behaviour. Regardless of the context, the focus remains the same. Applied Behaviour Analysis works on that, which is observable, measurable, and changeable through observation, data collection and use of methods which are supported through empirical evidence.

With a laser focus on behaviour, ABA meshes well with both psychology, whose study of the mind and its functions ultimately aims to assist with behaviour in a given context; and with psychiatry, whose study of the brain in a more physiological fashion sets its goal on treatment of abnormal behaviour, among other maladies. However, ABA contrasts the aforementioned sciences in that it does not seek to examine or treat that which can't be seen, proven, or measured objectively.

This allows ABA to be practiced by large numbers of professionals in a highly systematic and consistent manner. And so it has, in contexts from residential treatment facilities and outpatient programs to schools and homes. It is among this progression of environments that one may find the key to both quality of life from the psychological perspective and effectiveness from the behaviour analytical one.

Concentrating solely on the application of ABA to the treatment of Autism Spectrum Disorder, there are three main environments in which such treatment takes place: residential facilities, schools, and homes These settings vary in the both the level of restrictiveness and the presence of BCBAs supervising and/or running assessments and behaviour interventions.

Residential facilities range their level of restrictiveness, the highest of which are neurobehavioral stabilization lock-down units in which functional analyses are run and intensive in-patient behavior interventions are implemented, sometimes in combination with psychopharmacological interventions. These facilities treat individuals with the most severe behaviours and typically have BCBAs on staff and present at all times. While treatment provided in these settings can be very effective while the client resides there, upon returning home or being transferred to another setting, treatment effects are unlikely to generalize or be maintained for significant periods of time.

Less restrictive residential settings include campus type settings and community group homes. These settings typically have BCBAs on staff but present only part of the time as direct care staff carry out behaviour interventions written and monitored by the BCBAs. Caseloads and lack of training for direct care staff prevent behaviour interventions in these settings from being as effective as possible and changes to plans according to the data are typically not made in a timely manner.

The next tier of restrictiveness would be school settings; specifically out of district special education schools. In this environment, BCBAs may or may not be on staff and are typically available only for assessment and evaluation of progress with an intervention. Classroom staffs carry out behaviour interventions, usually with minimal training and supervision. These factors limit the effectiveness of treatment and the longevity of its effects.

In school district settings, a least restrictive setting, the client has access to a more full range of opportunities such as social events, extra-curricular activities, and an experience more closely resembling that of his/her neuro-typically developing peers. However, BCBAs are rarely on staff in school district settings and when they are, they are frequently tasked with carrying a caseload of an entire school or even an entire school district of several schools. This leaves little time for comprehensive assessments or thorough evaluation of ongoing behaviour interventions.

Another least restrictive setting for treatment to take place is in the

*Corresponding author: Rachel Sari, M.Ed., BCBA, Director of Clinica Services, Brett DiNovi \& Associates, LLC, 1000 Crawford Place, Suite 260, Mt. Laurel, NJ 08054, USA, Tel: 609-937-4714; E-mail: rachelesari@yahoo.com

Received September 16, 2015; Accepted December 05, 2015; Published December 12, 2015

Citation: Sari R (2016) Consultation in Applied Behavior Analysis: Making Least Restrictive Most Effective. J Psychiatry 19: 342 doi:10.4172/2378-5756.1000342

Copyright: (c) 2016 Sari R. This is an open-access article distributed under the terms of the Creative Commons Attribution License, which permits unrestricted use, distribution, and reproduction in any medium, provided the original author and source are credited 
client's own home or community. Certainly, it is extraordinarily rare for families to have a BCBA on staff in their homes, and arguably, one's presence would alter the environment, shifting it towards one that is more restrictive. This leaves it to a client's family to provide treatment, or to find community resources capable of providing treatment. It is in both this setting, and the school district setting mentioned earlier, where the role of the consultant becomes a crucial one, and potentially, the most effective.

Applied Behaviour Analysts working in consultation roles are able to provide services which are advantageous in schools, homes, and communities for several reasons. Among these are more manageable caseloads, networks of professional support, and flexibility of treatment options. However, possibly the most precious facet of consultation is the ability to train educational staff, families, and other caregivers to carry out behavioural strategies so that treatment effects can be generalized to multiple settings and maintained over longer periods of time, thus allowing for truly socially valid outcomes.
It is the unique ability of the consultant to use ABA with not only individuals with disabilities as their clients, but with their caretakers and stakeholders as well, that allows for any effective treatment to remain effective in the long term. A consultant may transfer stimulus control from themselves and/or direct care staff to other staff, family members, community members, natural stimuli in the environment, etc. In addition, the consultant continues to monitor data during this time to ensure that challenging behaviours remain low and functional equivalents remain high. Through this process, the consultant fades his or her own presence and increases the independence of the client. This opens a broader range of natural reinforcement to the client, thereby increasing the likelihood that the effects of intervention will remain.

For these reasons, one can see that through consultation, which takes place in the least restrictive of settings, we are able to implement the most effective treatment and gain the most durable and desirable results. 